\title{
Prolonged QTc affects short-term and long-term outcomes in patients with normal left ventricular function undergoing cardiac surgery
}

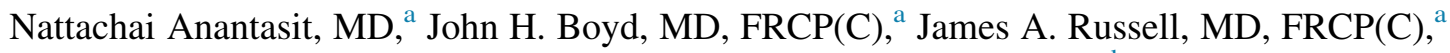 \\ Christopher D. Fjell, PhD, ${ }^{\mathrm{a}}$ Samuel V. Lichtenstein, MD, PhD, FRCSC, ${ }^{\mathrm{b}}$ and \\ Keith R. Walley, MD, $\operatorname{FRCP}(C)^{\mathrm{a}}$
}

\begin{abstract}
Objective: Although it is known that preoperative decreased left ventricular ejection fraction (LVEF) is a risk for morbidity and mortality after cardiac surgery, there are no reliable markers of risk in patients with preserved LVEF. This study examines whether a prolonged QTc interval is associated with adverse outcomes in patients with preoperative LVEF greater than $40 \%$ undergoing cardiac surgery.
\end{abstract}

\begin{abstract}
Methods: A retrospective chart review of patients who had cardiac surgery at St. Paul's Hospital in Vancouver, Canada, between 2004 and 2009, who had a preoperative LVEF greater than $40 \%$, was undertaken. We tested for association of preoperative prolonged QTc interval with mortality and morbidity using unadjusted and adjusted analyses.

Results: Five-hundred and fifty-five patients with a preoperative LVEF greater than $40 \%$ were included in the study; $496(89.4 \%)$ had cardiopulmonary bypass and the remainder were off pump. Preoperative prolonged QTc was associated with increased mortality at 30 days $(P<.01), 90$ days $(P<.01)$, and 8 years $(P<.01)$, and these results remained significant after adjusting for the clinical variables significantly associated with mortality (8-year odds ratio, 2.42; $95 \%$ confidence interval, $1.34-4.34 ; P=.003$ ). Similar results were found when the analysis was restricted to the more homogeneous group of patients undergoing on-pump coronary artery bypass (CABG, $\mathrm{n}=408)$. Prolonged QTc was also associated with prolonged intensive care unit stay $(P=.02)$, prolonged hospital stay $(P<.01)$, development of atrial arrhythmias $(P=.02)$, and low cardiac output syndrome (on-pump CABG, $P=.02$ ).

Conclusions: In patients undergoing cardiac surgery and a preoperative LVEF greater than $40 \%$, a prolonged QTc interval is associated with increased short-term and long-term mortality and increased perioperative morbidity, and therefore should be considered when assessing risk preoperatively. (J Thorac Cardiovasc Surg 2014;147:1627-33)
\end{abstract}

The heart rate-corrected QT interval (QTc) in the resting 12-lead electrocardiogram (ECG) provides important prognostic information on several conditions. QTc interval prolongation is associated with increased mortality in coronary heart disease and with increased ventricular arrhythmias and sudden cardiac death in diabetic and elderly patients. ${ }^{1-3}$ At least 3 studies have evaluated the

\footnotetext{
From the Centre for Heart Lung Innovation, ${ }^{a}$ Division of Cardiovascular Surgery, ${ }^{\mathrm{b}}$ University of British Columbia, St. Paul's Hospital, Vancouver, British Columbia, Canada.

This project was funded by Canadian Institutes of Health Research and Heart and Stroke Foundation. J. Boyd is a Michael Smith Foundation for Health Research and National Sanitorium Association Scholar. K. Walley is a Michael Smith Foundation for Health Research Distinguished Scholar. N. Anantasit is a Mahidol University Scholar.

Disclosures: J.A. Russell has acted as a consultant and owns equity in Sirius Genomics, Inc, has consulted for Ferring Pharmaceuticals, and has received grant support from Ferring Pharmaceuticals. S.V. Lichtenstein owns equity in Kardium, Inc (Can) and Ikemed Technologies, Inc (Can). All other authors have nothing to disclose with regard to commercial support.

Received for publication May 27, 2013; revisions received Nov 12, 2013; accepted for publication Nov 22, 2013; available ahead of print Jan 31, 2014.

Address for reprints: Keith R. Walley, MD, FRCP(C), Centre for Heart Lung Innovation, 1081 Burrard St, Vancouver, British Columbia, Canada, V6Z 1Y6 (E-mail: keith.walley@hli.ubc.ca).

$0022-5223 / \$ 36.00$

Copyright (c) 2014 by The American Association for Thoracic Surgery

http://dx.doi.org/10.1016/j.jtcvs.2013.11.043
}

association between prolonged QTc and postoperative outcomes after cardiac surgery. ${ }^{4-6}$ In all studies, prolonged preoperative QTc ( $>440$ milliseconds) was associated with increased mortality. Vrtovec and colleagues ${ }^{4}$ restricted their study to patients who had a left ventricular ejection fraction (LVEF) less than $40 \%$, whereas Kinoshita and colleagues ${ }^{5}$ and Foroughi and colleagues ${ }^{6}$ studied patients with any LVEF. Kinoshita and colleagues ${ }^{5}$ found that prolonged QTc interval is a predictor of all-cause mortality and sudden cardiac death over 5 years of follow-up in patients undergoing off-pump coronary surgery. However, whether preoperative prolonged QTc is associated with early and long-term postoperative adverse outcomes is unclear in cardiac surgical patients with an LVEF greater than $40 \%$, who are typically considered low risk. Our hypothesis was that prolonged QTc is associated with increased postoperative morbidity and mortality in patients undergoing cardiac surgery who have a preoperative LVEF greater than $40 \%$.

\section{PATIENTS AND METHODS \\ Patients}

Patients admitted to the cardiac surgical intensive care unit (ICU) at St. Paul's Hospital in Vancouver, Canada, between January 2004 and December 2009 were recruited to the study $(n=834)$. The primary 


$$
\begin{aligned}
& \text { Abbreviations and Acronyms } \\
& \begin{aligned}
\text { CABG } & =\text { coronary artery bypass graft } \\
\text { CI } & =\text { confidence interval } \\
\text { ECG } & =\text { electrocardiogram } \\
\text { eGFR } & =\text { estimated glomerular filtration rate } \\
\text { ICU } & =\text { intensive care unit } \\
\text { LCOS } & =\text { low cardiac output syndrome } \\
\text { LVEF } & =\text { left ventricular ejection fraction } \\
\text { OR } & =\text { odds ratio } \\
\text { QTc } & =\text { corrected QT interval } \\
\text { STS } & =\text { Society of Thoracic Surgeons }
\end{aligned}
\end{aligned}
$$

inclusion criteria were availability of a preoperative ECG within 3 months of surgery and preoperative LVEF greater than $40 \%(n=691)$. Exclusion criteria included preoperative atrial fibrillation, a preoperatively implanted pacemaker, patients who had prolonged QRS duration $(\geq 120$ milliseconds), and use of QT-prolonging drugs including amiodarone, procainamide, or antipsychotic drugs; leaving 555 patients for this study. In British Columbia, there is a single payor government universal health plan into which all citizens are enrolled while living in the province. Patients were censored on disenrollment from the health plan if they moved outside the province. Provincial vital statistics were used to determine the date of death. The research ethics boards of participating institutions approved this study and written informed consent was obtained from all patients or their authorized representatives. The institutional review board at St. Paul's Hospital and the University of British Columbia approved the study.

\section{Electrocardiographic Measurements}

Standard 12-lead resting ECG tracings at $25 \mathrm{~mm} / \mathrm{s}$ paper speed and $10 \mathrm{~mm} / \mathrm{mV}$ amplitude were performed before surgery using a MAC-5500 digital ECG system (GE Medical Systems). ECGs were analyzed using a Marquette 12SL ECG analysis program (GE Medical Systems). Every hard copy ECG was also reinterpreted by a cardiologist. The QT measurement was made in leads II and V5 or V6, and the longest value was used. QT interval was corrected for heart rate (QTc) using the Bazett formula. The QT interval was measured from the beginning of the earliest onset of the QRS complex to the end of the $T$ wave. The end of the T wave was defined as the return of the descending limb to the isoelectric line. When a discrete $U$ wave was present after the $T$ wave, the $T$ wave offset was defined as the nadir between the $T$ and $U$ waves. When the $T$ and $\mathrm{U}$ waves were fused, the $\mathrm{U}$ component was calculated as the $\mathrm{T}$ wave. Prolonged QTc was defined by using gender-specific QT categories (males, QTc $\geq 450$ milliseconds; females, QTc $\geq 470$ milliseconds).

\section{Primary and Secondary Outcomes}

The primary outcome variables were 30-day, 90-day, and 8-year mortality. The secondary outcome variables measured during the perioperative hospitalization were prolonged ICU stay defined as a stay in the cardiac ICU more than 48 hours, ${ }^{7}$ prolonged hospital stay defined as greater than 14 days, ${ }^{8}$ development of atrial arrhythmias, and development of low cardiac output syndrome (LCOS).

New-onset postoperative atrial arrhythmias were defined as any episode of atrial fibrillation or flutter within 30 days after surgery that required treatment. LCOS was diagnosed if (1) patients required an intra-aortic balloon pump to be weaned from cardiopulmonary bypass or in the ICU because of hemodynamic compromise, or (2) the patient required inotropic drug(s) to maintain systolic blood pressure greater than $90 \mathrm{~mm} \mathrm{Hg}$ and cardiac index greater than $2.2 \mathrm{~L} / \mathrm{min} / \mathrm{m}^{2}$ for at least 30 minutes in the
ICU and excluding patients who received less than $4 \mu \mathrm{g} / \mathrm{kg}$ of dopamine to increase renal perfusion or patients who received vasoconstrictor drugs to increase systemic vascular resistance. ${ }^{9,10}$

\section{Baseline and Perioperative Measurements}

Baseline characteristics of the patients were measured at study enrollment including age, gender, preexisting conditions (hypertension, diabetes, and myocardial ischemia, based on patients' recorded past medical history), current medications, LVEF, electrolytes, serum creatinine, and an estimated glomerular filtration rate (eGFR) using the Modification of Diet in Renal Disease Study equation. The impaired renal function was defined as eGFR less than $60 \mathrm{~m} / / \mathrm{min} / 1.73 \mathrm{~m}^{2}{ }^{11}$ The operative variables recorded were whether or not on cardiopulmonary bypass pump, cardiopulmonary bypass times, aortic crossclamp times, the cardioplegia temperature, type of cardioplegia, and urgency of operation (urgent indicating an operation within 48 hours). New-onset postoperative atrial arrhythmias were recorded if patients had atrial arrhythmias from a 12-lead ECG in the hospital computer system.

\section{Statistical Analysis}

Statistical analysis was performed using R, version 2.8.1 (www.Rproject.org) and SPSS, version 16 (SPSS, Inc; Chicago, Ill) statistical software packages. Patients who had prolonged QTc were compared with patients with normal QTc and tested for differences in baseline characteristics and adverse outcomes using the Mann-Whitney $U$ statistic test for continuous data or a chi-squared test for categorical data. Normally distributed continuous variables were summarized by mean and standard deviation, whereas nonnormally distributed continuous variables were summarized by median and interquartile ranges. Categorical variables were expressed as the number of patients and percentage. The Pearson correlation coefficient was calculated for the relationship (continuous) between the QTc interval and continuous variables of baseline and adverse outcome data. Logistic regression was used to measure the association between the clinical variables and mortality. Risk factors determined to be clinically significant a priori on the bivariable analyses were identified as candidate variables for the multivariable model. Survival analysis was compared between 2 groups using the log-rank test. Cox regression analysis was used to test for differences in survival and included those baseline variables from the univariate analysis with $P<.05$. In view of the heterogeneity of this cardiac surgery cohort, the analysis was repeated in the largest subset of patients; those undergoing coronary artery bypass surgery using cardiopulmonary bypass. Observed and predicted mortality risk were compared using Society of Thoracic Surgeons (STS) risk scores (version 2.73). ${ }^{12}$ In all comparisons, $P<.05$ was considered statistically significant.

\section{RESULTS \\ Baseline Characteristics}

Of 555 patients who had a preoperative LVEF greater than $40 \%$ and underwent cardiac surgery, $496(89.4 \%)$ had cardiopulmonary bypass; $465(83.8 \%)$ underwent cardiopulmonary bypass with coronary artery bypass graft surgery (CABG), $14(2.5 \%)$ patients underwent valve surgery, $66(11.9 \%)$ patients underwent combined valve plus $\mathrm{CABG}$, and $10(1.8 \%)$ patients underwent other cardiac surgery. The preoperative QTc interval was normally distributed. The mean of the preoperative QTc interval was $427 \pm 35$ milliseconds, preoperative LVEF was $55 \pm 8 \%$, and the mean age was $67 \pm 10$ years. Eighty-four $(15.1 \%)$ patients had a prolonged QTc interval 
(Table 1 ). Mortality was $1.4 \%$ at 30 days, $3.1 \%$ at 90 days, and $10.8 \%$ at 8 years after surgery. Prolonged ICU stay occurred in $13.3 \%$, prolonged hospital stay occurred in $8.5 \%$, new-onset atrial arrhythmias developed in $7.7 \%$ with a median time to onset of 3.5 days (range, 0 to 18 days) and LCOS developed in $21.1 \%$; the cardioplegia temperature, type of cardioplegia, and cardiopulmonary bypass were not statistically significantly associated with patients who had developed LCOS. Three patients required the intra-aortic balloon pump after surgery. Patients who had a prolonged QTc were significantly older (72 vs 67 years, $P<.01)$ and had a lower LVEF $(50 \%$ vs $57 \%$, $P<.01)$ than patients who had a normal QTc. There were no other significant differences between the groups (Table 1).

\section{Primary Outcome}

Kaplan-Meier survival curves (Figure 1) demonstrated that patients with a prolonged QTc interval had significantly higher 30-day, 90-day, and 8-year mortality than patients with a normal QTc interval $(P<.05)$. Cox regression analysis to adjust for the clinical variables significantly associated with mortality outcome (age, LVEF, prolonged QTc, urgent operation, impaired renal function, and type of operation) confirmed that patients with prolonged QTc had higher mortality (30-day odds ratio [OR], 9.09; 95\% confidence interval $[\mathrm{CI}], 1.54-53.61 ; P=.015 ; 90$-day OR, 7.69; 95\% CI, 2.41-24.55; $P=.001 ; 8$-year OR, $2.42 ; 95 \%$ CI, $1.34-4.34 ; P=.003)$ than patients with normal QTc. To determine whether there was an increase in mortality as QTc increased to more than 400 milliseconds, we compared patients in the following subgroups: less than 400, 401-420, 421-440, 441-460, 461-480, 481-500, and more than 500 milliseconds. Figure 2 shows that, although all levels of prolonged QTc were associated with increased mortality, there was a marked increase in mortality from less than $5 \%$ to greater than $25 \%$ as QTc increased.

\section{Secondary Outcomes}

Patients with a prolonged QTc also had significantly increased incidences of prolonged ICU stay $(P=.02)$ and hospital stay $(P<.01)$ (Table 2$)$. Multiple logistic regression analysis to adjust for the clinical variables significantly associated with mortality (age, LVEF, prolonged QTc, urgent operation, impaired renal function, and type of operation) confirmed that patients with prolonged QTc had a trend toward more frequent prolonged ICU stay (OR, 1.59; 95\% CI, 0.91-3.06; $P=.092)$ and had more frequent prolonged hospital stay (OR, 2.36; $95 \%$ CI, 1.16-4.78; $P=.018)$. Patients who had prolonged QTc interval had significantly more atrial arrhythmias than patients with normal QTc $(13.1 \%$ vs $6.8 \%, P=.04$; Table 2). To determine whether there was an increase in
TABLE 1. Baseline characteristics of patients who had cardiac surgery and who had a left ventricular ejection fraction greater than $40 \%$

\begin{tabular}{|c|c|c|c|}
\hline Characteristic & $\begin{array}{c}\text { Normal } \\
\text { QTe }(n=471)\end{array}$ & $\begin{array}{c}\text { Prolonged } \\
\text { QTe }(\mathbf{n}=84)\end{array}$ & $\boldsymbol{P}$ \\
\hline Age, y & $67(59-73)$ & $72(63-79)$ & $<.01 *$ \\
\hline Race: white, n (\%) & $429(91.1)$ & $80(95.2)$ & .28 \\
\hline Gender: male, n (\%) & $380(80.7)$ & $71(84.5)$ & .45 \\
\hline Body mass index, $\mathrm{kg} / \mathrm{m}^{2}$ & $27.5(25-31)$ & $27(25-31)$ & .76 \\
\hline Preoperative LVEF, \% & $57(50-60)$ & $50(45-60)$ & $<.01 *$ \\
\hline $\begin{array}{l}\text { Preoperative QTc interval, } \\
\text { milliseconds }\end{array}$ & $416(402-432)$ & $480(463-500)$ & $<.01^{*}$ \\
\hline Preoperative $\mathrm{Na}, \mathrm{mmol} / \mathrm{L}$ & $140(138-141)$ & $140(137-141)$ & .86 \\
\hline $\begin{array}{l}\text { Preoperative } \mathrm{K}, \\
\quad \text { mean } \mathrm{mmol} / \mathrm{L} \pm \mathrm{SD}\end{array}$ & $4.22( \pm 0.45)$ & $4.17( \pm 0.43)$ & .58 \\
\hline $\begin{array}{l}\text { Preoperative } \mathrm{Mg}, \\
\quad \text { mean } \mathrm{mmol} / \mathrm{L} \pm \mathrm{SD}\end{array}$ & $1.06( \pm 0.25)$ & $1.00( \pm 0.10)$ & .50 \\
\hline \multicolumn{4}{|l|}{ Preexisting condition, $\mathrm{n}(\%)$} \\
\hline Hypertension & 370 (78.6) & $70(83.3)$ & .38 \\
\hline Smoker & $67(14.2)$ & $12(14.3)$ & 1.00 \\
\hline Diabetes mellitus & $128(27.2)$ & $28(33.3)$ & .29 \\
\hline Dyslipidemia & $366(77.7)$ & $67(79.8)$ & .77 \\
\hline Impaired renal function & $79(16.8)$ & $31(36.9)$ & $<.01^{*}$ \\
\hline End-stage renal disease & $8(1.7)$ & $2(2.4)$ & .66 \\
\hline Congestive heart failure & 365 (77.5) & $66(78.6)$ & .83 \\
\hline History of MI & $56(11.9)$ & $14(16.7)$ & .29 \\
\hline \multicolumn{4}{|l|}{ Medications, n (\%) } \\
\hline Aspirin & $395(83.9)$ & $72(85.7)$ & .75 \\
\hline Clopidogrel & $62(13.2)$ & $9(10.7)$ & .60 \\
\hline ACE inhibitor & $182(38.6)$ & $32(38.1)$ & 1.00 \\
\hline$\beta$-Blocker & $324(68.8)$ & $48(57.1)$ & $.04 *$ \\
\hline Calcium blocker & $122(25.9)$ & $21(25.0)$ & 1.00 \\
\hline Statin & 309 (65.6) & $46(54.8)$ & .06 \\
\hline \multicolumn{4}{|l|}{ Perioperative data, $\mathrm{n}(\%)$} \\
\hline Urgent operation & $65(13.8)$ & $15(17.8)$ & .32 \\
\hline On CPB pump & 419 (88.9) & 77 (91.7) & .57 \\
\hline Bypass time, min & $83(63-101)$ & $87(62-110)$ & .29 \\
\hline Aortic crossclamp time, min & $64(42-81)$ & $65(41-87)$ & .62 \\
\hline
\end{tabular}

Data are median (interquartile range) for continuous variables. QTc, Corrected QT interval; $L V E F$, left ventricular ejection fraction; $S D$, standard deviation; $M I$, myocardial ischemia; $C P B$, cardiopulmonary bypass. ${ }^{*} P<.05$.

the incidence of atrial arrhythmias as QTc increased to more than 400 milliseconds, we compared patients in the following subgroups: QTc less than 400, 401-420, 421-440, 441-460, 461-480, 481-500 and more than 500 milliseconds. Risk of atrial arrhythmia increased with any prolongation of QTc but a much higher risk was observed with QTc greater than 500 milliseconds (Figure 2). Patients with a prolonged QTc also had a trend toward increased incidence of perioperative LCOS (Table 2).

Increased mortality in patients with a prolonged QTc was not accounted for by the increase in atrial arrhythmias in these patients. Table 3 shows that patients with preoperative prolonged QTc who did not develop atrial arrhythmias still had an 11-fold, 6-fold, and 3-fold increase in risk of 30-day, 90-day, and 8-year mortality, respectively, compared with 


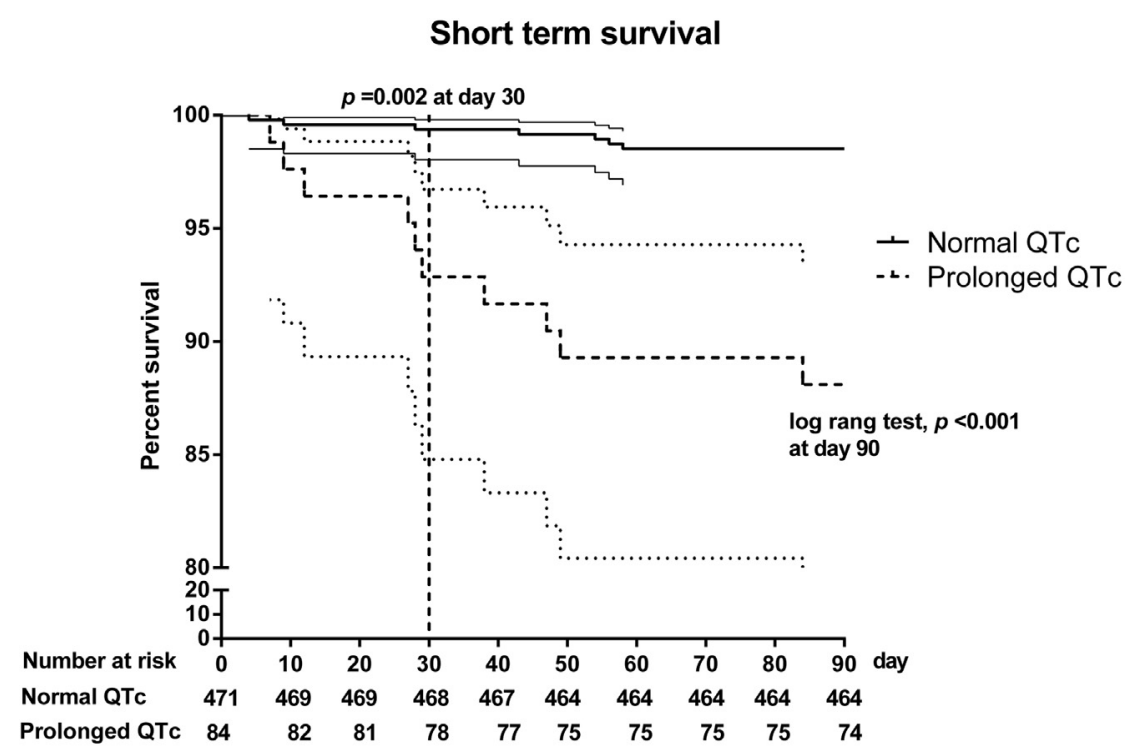

\section{Long term survival}

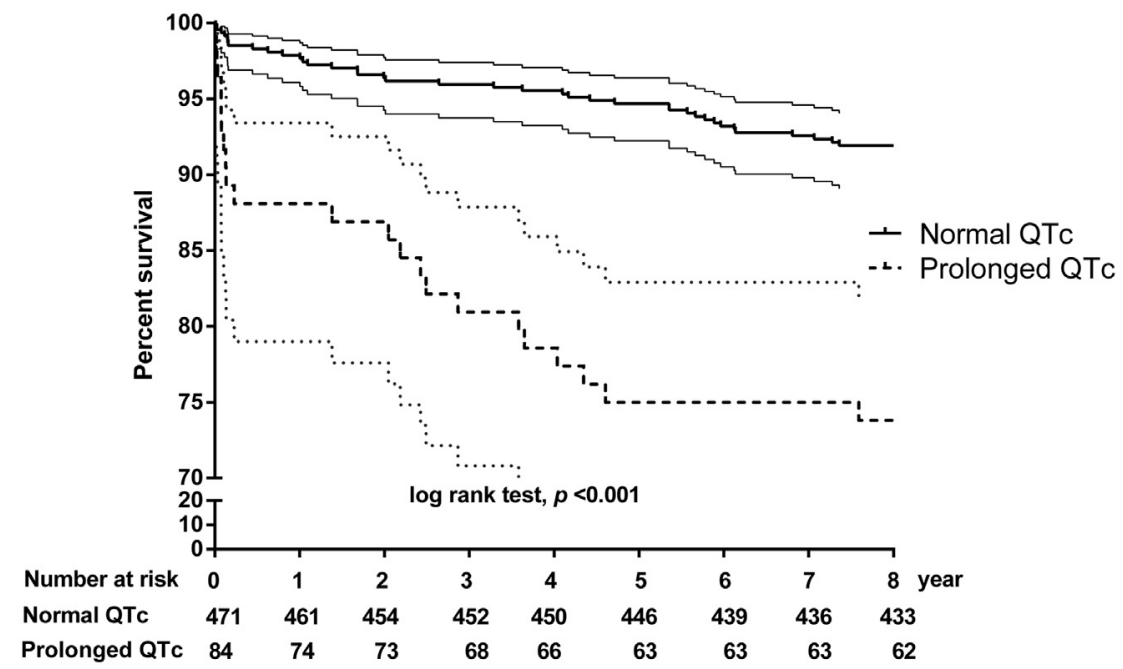

FIGURE 1. Kaplan-Meier survival curves for patients who underwent cardiac surgery and who had a left ventricular ejection fraction greater than $40 \%$. $Q T c$, Corrected QT interval.

patients who had normal QTc $(P<.01$ for each). In addition, patients who had prolonged QTc who did not develop atrial arrhythmias had more frequent prolonged ICU stay $(P=.039)$ and hospital stay $(P<.01)$ than patients with a normal QTc interval (Table 3).

\section{Homogeneous Subgroup}

The overall cohort of patients included the following procedures: on-pump and off-pump CABG, valve, combined valve and CABG, and other surgery. To confirm that our observations were not due to this mixture, we repeated the analysis for the largest homogenous group of patients, that is, patients who underwent on-pump CABG $(n=408)$. Baseline characteristics of on-pump CABG patients were similar to all patients. In this large, more homogeneous subgroup, patients with preoperative prolonged QTc had increased mortality at 30 days, 90 days, and 8 years (Table 2). These patients also had an increased incidence of prolonged hospital stay (Table 2). In addition, patients with prolonged QTc developed more atrial arrhythmias $(P=.04$, Table 2) and had a higher incidence of $\operatorname{LCOS}(P=.02$, Table 2) compared with patients who had normal QTc. We compared observed and STS predicted mortality risk in on-pump CABG patients. The observed and predicted STS mortalities matched for those patients with a normal QTc interval ( 0.8 vs 0.5 ). However, the observed mortality greatly exceeded the predicted STS risk model in patients who had preoperative prolonged QTc interval (7.27 vs 0.76). 

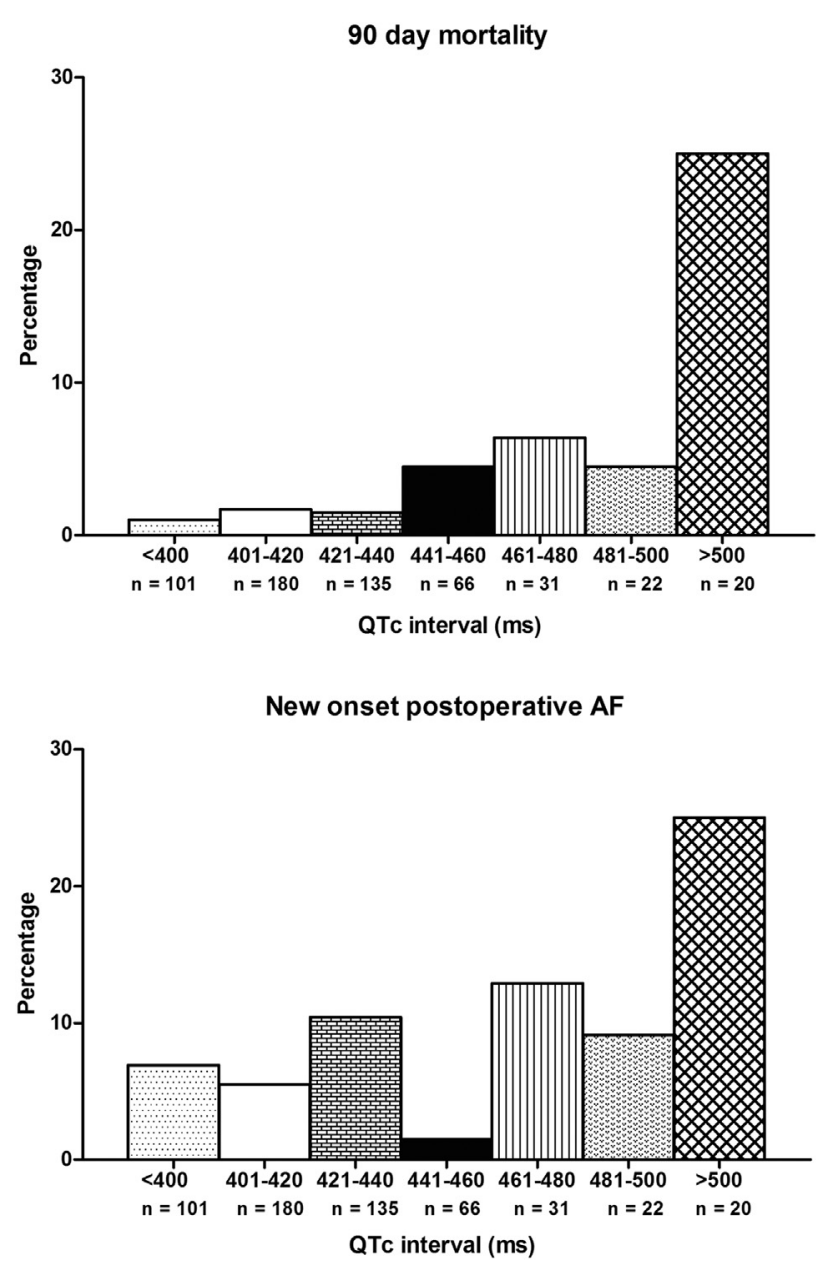

FIGURE 2. Incidence of 90-day mortality and new-onset postoperative atrial fibrillation $(A F)$ according to the preoperative QTc interval of patients who had cardiac surgery and who had a left ventricular ejection fraction greater than $40 \%$. QTc, Corrected QT interval.

\section{COMMENT}

The major finding of this study is that in patients who have cardiac surgery and a preoperative LVEF greater than $40 \%$ (generally considered low risk), prolonged QTc measured preoperatively is a risk for significantly increased 30-day, 90-day, and 8-year mortality and increased perioperative complications of atrial arrhythmia and LCOS. These findings remained in a repeat analysis of a more homogeneous subset; patients who underwent on-pump cardiopulmonary bypass with coronary artery graft surgery. Although previous studies have analyzed the risks associated with prolonged QTc interval after cardiac surgery; definitions of prolonged QTc varied ${ }^{4,6}$ and patients were restricted to those with heart failure (ie, decreased LVEF) ${ }^{4}$ or those who underwent off-pump surgery. ${ }^{5}$ By focusing on patients with LVEF greater than $40 \%$, we selected patients generally deemed to be low risk yet still found highly significant adverse mortality and morbidity outcomes associated with prolonged QTc. This adds to knowledge from previous studies demonstrating that prolonged QTc is an important risk factor. ${ }^{4-6,13}$

Modern surgical techniques and reduced cardiopulmonary bypass times have resulted in excellent outcomes for cardiac surgeries including $\mathrm{CABG}$ and valvular surgeries. Nonetheless, there remains a $1 \%$ to $2 \%$ risk of death even among patients without recognized risk factors such as end-stage renal failure, advanced heart failure, or older age. ${ }^{14,15}$ It is important to identify patients who have increased risk not only in the interest of their informed consent but also to tailor management such as duration of high acuity monitoring and care as well as pharmacologic therapy for prevention of arrhythmias. The preoperative ECG is inexpensive and in this study, we found that it was of significant usefulness in predicting which otherwise low-risk patients (with LVEF greater than $40 \%$ ) were at increased risk of death, postoperative atrial arrhythmias, and LCOS.

The QTc interval reflects the cardiac depolarization and repolarization times in 1 heart cycle ${ }^{16}$ and, in population surveys, prolonged QTc interval is associated with an increased risk of atrial-ventricular arrhythmias and ventricular systolic and diastolic dysfunction. ${ }^{17,18}$ Previous studies have shown an association between prolonged QTc and adverse postoperative outcomes including increased mortality. ${ }^{4-6}$ However, this association could have been confounded by the common coexistence of prolonged QTc with depressed left ventricular function as expressed by decreased LVEF. So it has not been clear whether prolonged QTc is an independent predictor of adverse outcomes after cardiac surgery. The STS risk model was developed to estimate the risk of operative mortality, based on multiple risk factors, to guide surgeons and cardiologists caring for patients undergoing isolated CABG, valve replacement, and CABG plus valve replacement. However, preoperative QTc interval is not one of preoperative variables in the STS risk model. Several studies have shown that observed mortality can be significantly higher than predicted STS mortality in the setting of other comorbidities. ${ }^{19,20}$ Our results add to this knowledge by finding that predicted STS mortality risk underestimates mortality in patients with preoperative prolonged QTc interval. Thus, risk assessment should additionally consider QTc when planning perioperative management.

Our study is novel for several reasons. First, we found an association between prolonged QTc and increased short-term and long-term mortality in patients undergoing cardiac surgery who had an LVEF greater than $40 \%$. Second, we found that prolonged QTc was associated with increased incidence of prolonged hospital stay, increased risk of postoperative atrial arrhythmias, and 
TABLE 2. Postoperative outcomes of patients who had cardiac surgery and who had a left ventricular ejection fraction greater than $40 \%$ (all patients, $n=555)$ and largest homogeneous group; on-pump CABG $(n=408)$

\begin{tabular}{|c|c|c|c|c|c|c|}
\hline \multirow[b]{2}{*}{ Outcome } & \multicolumn{3}{|c|}{ All patients $(n=555)$} & \multicolumn{3}{|c|}{ On-pump CABG $(n=408)$} \\
\hline & Normal QTc $(n=471)$ & Prolonged QTc $(n=84)$ & $\boldsymbol{P}$ & Normal QTe $(n=353)$ & Prolonged QTc $(\mathrm{n}=55)$ & $\boldsymbol{P}$ \\
\hline 30-d mortality, n (\%) & $3(0.6)$ & $5(6)$ & $<.01 *$ & $1(0.3)$ & $2(3.6)$ & .05 \\
\hline 90-d mortality, n (\%) & $7(1.5)$ & $10(11.9)$ & $<.01 *$ & $3(0.8)$ & $4(7.3)$ & $<.01 *$ \\
\hline 1-y mortality, n (\%) & $10(2.1)$ & $10(11.9)$ & $<.01 *$ & $4(1.1)$ & $4(7.3)$ & $<.01 *$ \\
\hline 2-y mortality, n (\%) & $17(3.6)$ & $11(13.1)$ & $<.01 *$ & $9(2.5)$ & $4(7.3)$ & .06 \\
\hline 5-y mortality, n (\%) & $25(5.3)$ & $21(25)$ & $<.01 *$ & $14(3.9)$ & $11(20)$ & $<.01 *$ \\
\hline 8-y mortality, n (\%) & $38(8.1)$ & $22(26.2)$ & $<.01 *$ & $24(6.8)$ & $12(21.8)$ & $<.01^{*}$ \\
\hline Length of ICU stay, h & $24(23-28)$ & $26(24-46.5)$ & $<.01 *$ & $24(22-27)$ & $25(23.5-27)$ & .11 \\
\hline Length of hospital stay, $\mathrm{d}$ & $5(5-7)$ & $6(5-10)$ & $<.01 *$ & $5(4.5-7)$ & $6(5-8)$ & $.04 *$ \\
\hline Prolonged ICU stay, n (\%) & $56(11.9)$ & $18(21.4)$ & $.02 *$ & $24(6.8)$ & $5(9.1)$ & .57 \\
\hline Prolonged hospital stay, n (\%) & $31(6.6)$ & $16(19.0)$ & $<.01 *$ & $15(4.2)$ & $7(12.7)$ & $.02 *$ \\
\hline \multicolumn{7}{|l|}{ Complications, $\mathrm{n}(\%)$} \\
\hline $\mathrm{AF}$ & $32(6.8)$ & $11(13.1)$ & $.04 *$ & $15(4.2)$ & $6(10.9)$ & $.04 *$ \\
\hline LCOS & $93(19.7)$ & $24(28.6)$ & .08 & $73(20.7)$ & $19(34.5)$ & $.02 *$ \\
\hline
\end{tabular}

$\overline{C A B G}$, Coronary artery bypass graft; $Q T c$, corrected QT interval; $I C U$, intensive care unit; $A F$, new-onset postoperative atrial fibrillation or flutter; $L C O S$, low cardiac output syndrome. $* P<.05$

increased incidence of LCOS. Third, we found that when QTc is greater than 400 milliseconds, mortality is increased and then markedly increased in patients who have QTc greater than 500 milliseconds. Fourth, we found that preoperative prolonged QTc adds additional information to STS mortality risk estimates.

Our data suggest that preoperative ECG screening for the presence of a prolonged QTc interval is a useful indicator of the risk of short-term and long-term mortality and perioperative morbidity in otherwise low-risk patients scheduled for cardiac surgery. Interventions in patients who have prolonged QTc may depend on the cause of the prolonged QTc, such as cessation or dose reduction of antiarrhythmic (eg, amiodarone, procainamide, or disopyramide), antipsychotic (eg, haloperidol or chlorpromazine), antibiotic (eg, macrolides or fluoroquinolones), or antihistamine (eg, terfenadin) medications known to prolong QTc. ${ }^{21}$ Perioperative normalization of electrolytes, especially potassium and magnesium, may assume greater importance in these patients in decreasing the risks of prolonged QTc. Barring contraindications such as reactive airway disease, $\beta$-blocker therapy begun at least 1 week before surgery has been shown to be efficacious in preventing postoperative atrial arrhythmias. ${ }^{22}$

This study has limitations. It is a single-center retrospective study and so subject to the possibility that QTc is simply a surrogate marker of the true cause of mortality and postoperative atrial arrhythmias and LCOS. The strong association between preoperative prolonged QTc and postoperative atrial arrhythmias in many studies supports a causal link, at least for atrial arrhythmias. With respect to generalizability of the study, hospital mortality in this study is $2.9 \%$, higher than expected in the literature. ${ }^{9,23}$ This may be due to inclusion of patients undergoing both coronary bypass and valvular repair/replacement. This broader inclusion may increase the applicability of the study to general cardiac surgical units. In addition, the results from the largest homogeneous subgroup, those patients undergoing on-pump CABG, was similar to the entire cohort suggesting that these results are not due to heterogeneity of this cohort. However, the numbers of patients who underwent valve and combined surgery are

TABLE 3. Comparison of outcomes of patients who had cardiac surgery and who had a left ventricular ejection fraction greater than $40 \%$, and who did not develop postoperative atrial arrhythmias

\begin{tabular}{lcrrr}
\hline \multicolumn{1}{c}{ Outcome } & Normal QTc $(\mathbf{n}=\mathbf{4 3 9})$ & Prolonged QTc $(\mathbf{n}=\mathbf{7 3})$ & $\boldsymbol{P}$ & $\mathbf{R R}(\mathbf{9 5} \% \mathbf{C I})$ \\
\hline 30-d mortality, n (\%) & $3(0.6)$ & $5(6.8)$ & $<.01^{*}$ & $2.31(1.01-5.65)$ \\
90-d mortality, n (\%) & $6(1.4)$ & $6(8.2)$ & $<.01^{*}$ & $1.73(1.02-3.05)$ \\
1-y mortality, n (\%) & $8(1.8)$ & $6(8.2)$ & $<.01^{*}$ & $1.52(1.02-2.39)$ \\
2-y mortality, n (\%) & $15(3.4)$ & $7(9.6)$ & $.02^{*}$ & $1.27(1.01-1.69)$ \\
5-y mortality, n (\%) & $21(4.8)$ & $17(23.3)$ & $<.01^{*}$ & $1.59(1.19-2.13)$ \\
8-y mortality, n (\%) & $33(7.5)$ & $18(24.7)$ & $<.01^{*}$ & $1.36(1.11-1.67)$ \\
Prolonged ICU stay, n (\%) & $47(10.7)$ & $14(19.2)$ & $.04 *$ & $1.13(1.01-1.30)$ \\
Prolonged hospital stay, n (\%) & $25(5.7)$ & $11(15.1)$ & $<.01 *$ & $1.25(1.02-1.56)$ \\
LCOS, n (\%) & $88(20.0)$ & $18(24.7)$ & .37 & $1.04(0.948-1.14)$ \\
\hline
\end{tabular}

$Q T c$, Corrected QT interval; $R R$, relative risk; $C I$, confidence interval; $I C U$, intensive care unit; $L C O S$, low cardiac output syndrome. $* P<.05$. 
still small. A larger sample size of these subgroups would have allowed analysis of other subgroups. The incidence of postoperative atrial arrhythmias was lower than in other studies. $^{24,25}$ We determined the postoperative atrial arrhythmias from 12-lead ECGs in the hospital system, which would have underestimated the true incidence. Our study used the Bazett formula to correct the QTc interval, which seems to overestimate the QTc interval at a faster heart rate than Hodges' linear correction formula. However, the Bazett formula is still the most commonly used in clinical practice and research.

\section{CONCLUSIONS}

Prolongation of the QTc interval in a preoperative ECG of patients undergoing cardiac surgery and who have an LVEF greater than $40 \%$ is a significant risk factor for short-term and long-term mortality, prolonged ICU and hospital stays, postoperative atrial arrhythmias, and LCOS. If the QTc exceeds 500 milliseconds, $25 \%$ of patients will develop postoperative atrial arrhythmias and $25 \%$ will not survive to hospital discharge. We recommend routine preoperative ECG screening in all patients and then optimization of potential contributors when prolonged QTc is observed.

The authors thanks the cardiothoracic surgeon team at St. Paul's Hospital, University of British Columbia, Vancouver, Canada for their contribution.

\section{References}

1. Dekker JM, Schouten EG, Klootwijk P, Pool J, Kromhout D. Association between QT interval and coronary heart disease in middle-aged and elderly men. Circulation. 1994;90:779-85.

2. Dekker JM, Crow RS, Hannan PJ, Schouten EG, Folsom AR. Heart rate-corrected QT interval prolongation predicts risk of coronary heart disease in black and white middle-aged men and women: the ARIC study. J Am Coll Cardiol. 2004;43:565-71.

3. Rossing P, Breum L, Major-Pedersen A, Sato A, Winding H, Pietersen A, et al. Prolonged QTc interval predicts mortality in patients with type 1 diabetes mellitus. Diabet Med. 2001;18:199-205.

4. Vrtovec B, Yazdanbakhsh A, Pintar T, Collard SD, Gregoric ID, Radovancevic B. QTc interval prolongation predicts postoperative mortality in heart failure patients undergoing surgical revascularization. Tex Heart Inst J. 2006;33:3-8.

5. Kinoshita T, Asai T, Suzuki T, Matsubayashi K, Horie M. Time course and prognostic implications of QT interval in patients with coronary artery disease undergoing coronary bypass surgery. J Cardiovasc Electrophysiol. 2012;23: 645-9.

6. Foroughi M, Karkhaneh Yousefi Z, Majidi Tehrani M, Noori Foroutaghe A, Ghanavati A, Hassantash SA. Prolonged QT interval and coronary artery bypass mortality due to heart failure. Asian Cardiovasc Thorac Ann. 2009;17: 604-7.

7. Ettema RG, Peelen LM, Schuurmans MJ, Nierich AP, Kalkman CJ, Moons KG. Prediction models for prolonged intensive care unit stay after cardiac surgery: systematic review and validation study. Circulation. 2010;122:682-9.

8. Peterson ED, Coombs LP, Ferguson TB, Shroyer AL, DeLong ER, Grover FL, et al. Hospital variability in length of stay after coronary artery bypass surgery: results from the Society of Thoracic Surgeon's National Cardiac Database. Ann Thorac Surg. 2002;74:464-73.

9. Rao V, Ivanov J, Weisel RD, Ikonomidis JS, Christakis GT, David TE. Predictors of low cardiac output syndrome after coronary artery bypass. J Thorac Cardiovasc Surg. 1996;112:38-51.

10. Maganti MD, Rao V, Borger MA, Ivanov J, David TE. Predictors of low cardiac output syndrome after isolated aortic valve surgery. Circulation. 2005;112: I448-52.

11. National Kidney Foundation. K/DOQI clinical practice guidelines for chronic kidney disease: evaluation, classification and stratification. Am J Kidney Dis. 2002;39(Suppl 1):S1-266.

12. STS National Database Risk Calculator. http://www.sts.org/sections/ stsnationaldatabase/riskcalculator. Accessed January 15, 2013.

13. Macfarlane PW, Murray H, Sattar N, Stott DJ, Ford I, Buckley B, et al. The incidence and risk factors for new onset atrial fibrillation in the PROSPER study Europace. 2011;13:634-9.

14. Lok CE, Austin PC, Wang H, Tu JV. Impact of renal insufficiency on short- and long-term outcomes after cardiac surgery. Am Heart J. 2004;148:430-8.

15. Appoo J, Norris C, Merali S, Graham MM, Koshal A, Knudtson ML, et al. Long-term outcome of isolated coronary artery bypass surgery in patients with severe left ventricular dysfunction. Circulation. 2004;110:II-13-7.

16. Zabel M, Hohnloser SH, Behrens S, Li YG, Woosley RL, Franz MR. Electrophysiologic features of torsades de pointes: insights from a new isolated rabbit heart model. J Cardiovasc Electrophysiol. 1997;8:1148-58.

17. Wilcox JE, Rosenberg J, Vallakati A, Gheorghiade M, Shah SJ. Usefulness of electrocardiographic QT interval to predict left ventricular diastolic dysfunction. Am J Cardiol. 2011;108:1760-6.

18. Padmanabhan S, Silvet H, Amin J, Pai RG. Prognostic value of QT interval and QT dispersion in patients with left ventricular systolic dysfunction: results from a cohort of 2265 patients with an ejection fraction of $<$ or $=40 \%$. Am Heart J. $2003 ; 145: 132-8$

19. Kennedy JL, LaPar DJ, Kern JA, Kron IL, Bergin JD, Kamath S, et al. Does the society of Thoracic Surgeons risk score accurately predict operative mortality for patients with pulmonary hypertension? J Thorac Cardiovasc Surg. 2013;146: 631-7.

20. Qadir I, Salick MM, Perveen S, Sharif H. Mortality from isolated coronary bypass surgery: a comparison of society of Thoracic Surgeons and the EuroSCORE risk prediction algorithms. Interact Cardiovasc Thorac Surg. 2012;14:258-62.

21. Li EC, Esterly JS, Pohl S, Scott SD, McBride BF. Drug-induced QT-interval prolongation: considerations for clinicians. Pharmacotherapy. 2010;30:684-701.

22. Camm AJ, Kirchhof P, Lip GY, Schotten U, Savelieva I, Ernst S, et al. Guidelines for the management of atrial fibrillation: the Task Force for the Management of Atrial Fibrillation of the European Society of Cardiology (ESC). Europace. 2010; $12: 1360-420$.

23. Michalopoulos A, Tzelepis G, Dafni U, Geroulanos S. Determinants of hospital mortality after coronary artery bypass grafting. Chest. 1999;115:1598-603.

24. Creswell LL, Schuessler RB, Rosenbloom M, Cox JL. Hazards of post-operative atrial arrhythmias. Ann Thorac Surg. 1993;56:539-49.

25. Leitch JW, Thomson D, Baird DK, Harris PJ. The importance of age as a predictor of atrial fibrillation and flutter after coronary artery bypass grafting J Thorac Cardiovasc Surg. 1990;100:338-42. 